\section{THU0340 ADRENAL INSUFFICIENCY DURING GLUCOCORTICOID TREATMENT IN PATIENTS WITH POLYMYALGIA REUMATICA OR GIANT CELL ARTERITIS}

T. Laursen ${ }^{1}$, H. Locht ${ }^{1}$, B. Jensen ${ }^{1}$, S. Borresen ${ }^{2}$, U. Feldt-Rasmussen ${ }^{2}$, L. Hilsted ${ }^{3}$, E.M. Bartels ${ }^{4} .{ }^{1}$ Center for Rheumatology and Spine Diseases, Rigshospitalet; ${ }^{2}$ Department of Endocrinology, Rigshospitalet; ${ }^{3}$ Department of Clinical Biochemistry, Rigshospitalet; ${ }^{4}$ The Parker Institute, Department of Rheumatology, Copenhagen, Denmark

Background: Adrenal insufficiency secondary to long-term systemic glucocorticoid treatment is a well-recognized problem. However, the extent and prevalence of this phenomenon has not been thoroughly explored.

Objectives: To investigate the prevalence of adrenal insufficiency in patients with polymyalgia reumatica (PMR) and giant cell arteritis (GCA) during treatment with low doses of prednisolone $(<10 \mathrm{mg} /$ day) assessed by the adrenal response to a 250 microgram Synachten ${ }^{\circledR}$ test. To explore whether potential adrenal insuffiency was associated with duration of steroid treatment.

Methods: Outpatients were examined when prednisolone doses were between 2.5 and $10 \mathrm{mg} /$ day for $>6$ months. Adrenal function was evaluated after a 48-hour pause of prednisolone, using a $250 \mu \mathrm{g}$ Synachten $^{\circledR}(\mathrm{ACTH})$ test where plasma cortisol levels were measured at baseline and 30 minutes after Synacthen injection. Adrenal insufficiency was defined as plasma cortisol $<420 \mathrm{nmol} / \mathrm{l}$ after 30 minutes according to the validated Roche Elecsys ${ }^{\circledR}$ Cortisol II assay. Accummulated doses of prednisolone for the individual patients were calculated. A multiple regression analysis was used to test for an association between the plasma cortisol after 30 minutes and the accumulated dose of prednisolone.

Results: Forty-eight patients (35 women) completed the Synachten ${ }^{\circledR}$ test. Seven (14.6\%) patients exhibited adrenal insufficiency. Median age was 74 years (Range: 57-89 years). Median accumulated dose was $3.402 \mathrm{mg}$ (Range: $820-21.200 \mathrm{mg}$ ). Median plasma cortisol after 30 minutes was $562 \mathrm{nmol} / /$ (Range: $92-989 \mathrm{nmol} / /$ ). In patients with adrenal insufficiency, median plasma cortisol was $122 \mathrm{nmol} / \mathrm{l}$ (Range: 56-275 nmol//) at baseline and 207 (Range: 92-420 nmol/l) after 30 minutes. In patients without adrenal insufficiency, median plasma cortisol was 359 (Range: $9-710 \mathrm{nmol} / \mathrm{l}$ ) at baseline and $584 \mathrm{nmol} / \mathrm{l}$ (Range: $429-989 \mathrm{nmol} / \mathrm{l}$ ) after 30 minutes. Accumulated doses of prednisolone did not differ in patients with and without adrenal insufficiency $(p=0.49)$. Plasma cortisol after 30 minutes was not associated with accumulated dose of prednisolone (estimate $-0.01,95 \%$ $\mathrm{Cl}:-0.02$ to $5.39, \mathrm{p}=0.06$ ), when adjusting for sex and age.

Conclusions: latrogenic adrenal insufficiency was prevalent among patients with PMR or GCA treated with low dose prednisolone. Adrenal function was not associated with the accumulated dose of prednisolone, or the duration of steroid treatment. A Synacthen ${ }^{\circledR}$ test is a valuable tool to evaluate the adrenal function, when steroid treatment is tapered in these patients. Latent symptoms of adrenal insufficiency may explain why some patients are reluctant to discontinue steroid treatment even after the inflammatory condition has gone into remission. Disclosure of Interest: None declared

DOI: 10.1136/annrheumdis-2017-eular.6623

\section{THU0341 FLT3 LIGAND CONCENTRATIONS ARE ELEVATED IN ANCA-ASSOCIATED VASCULITIDES (AAV) AND ARE INFLUENCED BY IMMUNOSUPPRESSIVE THERAPY}

N. Venhoff, J. Thiel, R.E. Voll, A.C. Venhoff, U. Salzer. Department of Rheumatology and Clinical Immunology, University Medical Center Freiburg, Medical Faculty, University of Freiburg, Freiburg, Germany

Background: The cytokine Flt3 ligand is an important cofactor for early hematopoiesis by mainly driving the development of lymphoid and early B-cell precursors. In the periphery functions of Flt3 are more pleiotropic involving the differentiation of regulatory T-cells, dendritic cells as well as peripheral B-cells. Besides its well-known roles in hematological disorders and as an indicator of bone marrow (BM) output capacity, the possible involvement of FLT3 ligand in autoimmune disorders was only discovered recently.

Objectives: Our primary aim was to analyze if FLT3 ligand serum concentrations are affected in AAV patients. Secondary aims were to correlate the FLT3 ligand serum concentrations with clinical and laboratory parameters. And, since FLT3 ligand concentrations are elevated in different states of bone marrow failure, we also wanted to evaluate FLT 3ligand as a marker for treatment related BM toxicity in AAV patients.

Methods: We performed a cross sectional study using a sandwich ELISA to determine FLT3 ligand concentrations in the serum of 98 well characterized AAV patients (69 GPA, 20 MPA and 9 EGPA) and 144 healthy controls (HC). Statistical evaluation was done using Mann-Whitney or unpaired, two-tailed Student's t-test. Results: In patients with AAV, FLT3 ligand concentrations were significantly elevated $(207 \mathrm{pg} / \mathrm{ml}+/-116.2$ in AAV versus $142.5 \mathrm{pg} / \mathrm{ml}+/-65.98$ in $\mathrm{HC}$; $\mathrm{p}<0.0001)$. Disease specific analysis revealed significantly elevated FLT3 ligand concentrations in GPA $(217.6 \mathrm{pg} / \mathrm{ml}+/-123 ; \mathrm{p}<0.0001)$, but no significant differences for MPA and EGPA when compared to HC. FLT3 ligand concentrations did not correlate to serological or clinical markers of disease activity, however, overall disease activity was low in the studied cohort. To assess the influences of treatment regimen on FLT3 ligand concentrations, we focused our analysis on treatment histories of cyclophosphamide (CYC) and azathioprine (AZA) and grouped the patient cohort according to the cumulative CYC dose $(<$ or $>5 \mathrm{~g})$ and/or duration of AZA therapy ( $<$ or $>6$ months). AZA and CYC naïve patients $(n=10)$ showed FLT3 concentrations comparable to $\mathrm{HC}(121 \mathrm{pg} / \mathrm{ml}+/-42.3)$, but in patients with low dose CYC and short term AZA therapy FLT3 concentrations were significantly higher $(176.6 \mathrm{pg} / \mathrm{ml}+/-51.6)$, both compared to treatment naïve patients and $\mathrm{HC}(\mathrm{p}=0.0095$ vs. AZA/CYC naïve, $p=0.0105$ vs $\mathrm{HC})$. Intensified treatment was associated with even further increased concentrations of FLT 3 ligand with highest concentrations found in patients treated with $>5 \mathrm{~g}$ CYC cumulative dose or AZA treatment for $>6$ months $(263.4 \mathrm{pg} / \mathrm{ml}+/-172.6 ; \mathrm{p}=0.0024$ vs. AZA/CYC naïve, $\mathrm{p}<0.0001$ vs $\mathrm{HC})$.

Elevated FLT3 serum concentrations in ANCA-associated vasculitides

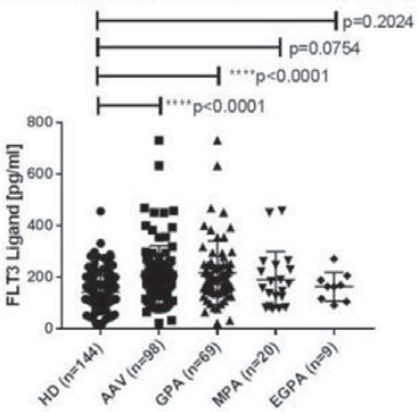

FLT3 ligand serum concentrations are influenced by therapy

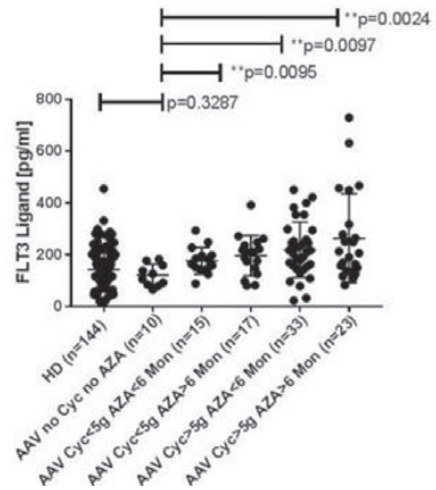

Conclusions: Flt3 ligand concentrations are elevated in patients with AAV, especially in patients with GPA. The elevation more likely reflects the therapeutic regimen and history than disease activity as we could show that patients with more intensive treatment including both CYC and AZA show higher FLT3 ligand serum levels when compared to patients with less intense therapy.

References:

[1] Ramos MI et al. FMS-related tyrosine kinase 3 ligand (Flt3L)/CD135 axis in rheumatoid arthritis. Arthritis Res Ther. 2013; 15:R209.

[2] Giri $\mathrm{N}$ et al. Immune status of patients with inherited bone marrow failure syndromes.Am J Hematol. 2015; 90:702-8.

Disclosure of Interest: None declared

DOI: 10.1136/annrheumdis-2017-eular.6349

\section{THU0342 UTILIZING "REAL LIFE" DATA IN ORDER TO EVALUATE THE ASSOCIATION BETWEEN GIANT CELL ARTERITIS AND AUTOIMMUNE THYROID DISEASE}

Y. Yavne ${ }^{1}$, S. Tiosano ${ }^{2}$, A. Watad ${ }^{2}$, D. Comaneshter ${ }^{3}$, Y. Shoenfeld ${ }^{2}$, A.D. Cohen ${ }^{3,4}, \mathrm{H}$. Amital ${ }^{5}$. ' Sackler Faculty of Medicine, Tel Aviv University, Tel Aviv; ${ }^{2}$ Department of Medicine 'B', Zabludowicz Center for Autoimmune Diseases, Sheba Medical Center, Tel Hashomer; ${ }^{3}$ Chief Physician's Office, Clalit Health Services, Tel Aviv; ${ }^{4}$ Siaal Research Center for Family Medicine and Primary Care, Faculty of Health Sciences, Ben Gurion University of the Negev, Beer Sheva; ${ }^{5}$ Department of Medicine 'B', Zabludowicz Center for Autoimmune Diseases, Sheba Medical Center, Tel Aviv, Israel

Background: In 1977, How et al. ${ }^{1}$ described the case of a simultaneous presentation of giant cell arteritis (GCA) and hypothyroidism. In the following decades, numerous studies have attempted to determine whether a significant interaction exists between GCA and autoimmune thyroid dysfunction, with conflicting results ${ }^{2-5}$.

Objectives: To evaluate whether a genuine association exists between GCA and autoimmune thyroid disease.

Methods: Utilizing the medical database of Clalit Health Services, we compared the proportion of autoimmune thyroid disease between patients with GCA and age- and gender-matched controls in a cross-sectional study. Univariate analysis was performed using Chi-square and student $t$-test and a multivariate analysis was performed using a logistic regression model.

Results: 5,663 GCA patients and 23,308 age- and gender-matched controls were 
included in the study. The proportion of hypothyroidism amongst GCA patients was increased in comparison with controls $(18.2 \%$ vs. $6.91 \%$, respectively, $p$-value $<0.001)$, as was hyperthyroidism $(2.56 \%$ and $1.19 \%$ respectively, $p$ value $<0.001$ ). After controlling for confounders, GCA demonstrated a robust independent association with hypothyroidism on multivariate logistic regression (OR 1.297, 95\% Cl 1.187-1.418, Table 1). In contrast, when a similar model was performed in order to assess the nature of the association between GCA and hyperthyroidism, it was found to be non-significant, with an OR of 1.097

Table 1. Multivariate logistic regression of covariates associated with hypothyroidism

\begin{tabular}{lccc}
\hline Characteristic & OR & $\mathrm{Cl}$ & $\mathrm{P}$ \\
\hline Age & 1.03 & $1.02-1.03$ & $<0.001$ \\
Gender (Female) & 3.32 & $2.93-3.79$ & $<0.001$ \\
BMI & 1.02 & $1.01-1.03$ & $<0.001$ \\
SES: & & & \\
$\quad$ Medium vs. Low & 1.42 & $1.28-1.58$ & $<0.001$ \\
$\quad$ High vs. Low & 1.63 & $1.45-1.83$ & $<0.001$ \\
$\quad$ GCA & 1.30 & $1.12-1.42$ & $<0.001$ \\
\hline
\end{tabular}

BMl: Body Mass Index, kg/m², SES: Socioeconomic status, GCA: Giant Cell Arteritis.

Conclusions: GCA patients have a higher proportion of hypothyroidism in comparison with matched controls. A significant association between GCA and hyperthyroidism was not found. Physicians treating GCA patients should consider screening for thyroid dysfunction on a regular basis.

References:

[1] How J, Bewsher PD, Walker W. Giant-cell arteritis and hypothyroidism. Br Med J. 1977;2(6079):99.

[2] Wiseman P, Stewart K, Rai GS. Hypothyroidism in polymyalgia rheumatica and giant cell arteritis. BMJ. 1989;298(6674):647.

[3] Bowness P, Shotliff K, Middlemiss A, Myles AB. Prevalence of hypothyroidism in patients with polymyalgia rheumatica and giant cell arteritis. Rheumatology. 1991;30(5):349-351.

[4] Duhaut $\mathrm{P}$, Bornet $\mathrm{H}$, Pinède $\mathrm{L}$, et al. Giant cell arteritis and thyroid dysfunction: multicentre case-control study. BMJ. 1999;318(7181):434-435.

[5] Myklebust G, Gran JT. A prospective study of 287 patients with polymyalgia rheumatica and temporal arteritis: clinical and laboratory manifestations at onset of disease and at the time of diagnosis. Rheumatology. 1996;35(11):11611168.

Disclosure of Interest: None declared

DOI: 10.1136/annrheumdis-2017-eular.2555

\section{THU0343 THE EFFECT OF ADALIMUMAB ON CLINICAL MANIFESTATIONS AND PRO-INFLAMMATORY CYTOKINES MILIEU IN PATIENTS WITH BEHCET'S DISEASE}

Y. Braun-Moscovici, Y. Tavor, D. Markovits, K. Toledano, A. Rozin, M.A. Nahir, A. Balbir-Gurman. Rheumatology Department, Rambam Health Care Campus, Rappaport Faculty of Medicine, Technion, Haifa, Israel

Background: Behcet's disease is a multisystemic chronic relapsing inflammatory disease, classified among the vasculitides. The aetiology of Behcet's disease is unknown. Several cytokines, among them TNF- $\alpha$, are involved in the pathogenesis of the disease.

Objectives: We aimed to assess efficacy and safety of Adalimumab (ADA) in patients with active Behcet's arthritis not responding to one or more DMARDS and to assess the impact of treatment on the cytokine milieu.

Methods: Eligible patients (pts) with active arthritis were enrolled in a 24 weeks single center prospective open-label study. Pts who relapsed within 12 weeks following ADA discontinuation could enter a 3 year extension study. The efficacy was assessed by 68 tender and 66 swollen joint count, patient visual analogue scale (VAS) for pain, physician overall disease activity VAS, health assessment questionnaire (HAQ), Behçet's Disease Current Activity Form (BDCAF), C reactive protein (CRP) and erythrocyte sedimentation rate (ESR). TNF- $\alpha$, IL-1 $1 \beta$, IL-6, INF- $\gamma$, $\mathrm{IL}-10$ and IL-17a were evaluated at baseline, after 24 and 48 weeks of treatment, by ProcartaPlex Human High Sensitivity - Immunoassay kit. Trough ADA serum levels and anti-drug antibodies were measured at baseline, week 24 and 48.

Results: Ten pts (6 females),age (mean, standard deviation -SD) 45 (8.4) years, with a disease duration of 11.6 (10) years, were enrolled and treated with subcutaneous ADA $40 \mathrm{mg}$ every 2 weeks for 24 weeks. The results are described

Abstract THU0344 - Table 1 in Table1. A statistically significant improvement was observed in swollen joint count, physician VAS and BDCAF and in IL-6 levels, but not in tender joint count or HAQ. Resolution of oral and urogenital ulcers was achieved in all pts. Significant reduction of pain was reported by $40 \%$ of pts. No relapse of uveitis or other disease manifestations occurred during the study. The reduction in IL-6 levels correlated with the physician VAS and BDCAF but not with HAQ. No correlation was found between change in IL-10 level and VAS pain. The levels of INF- $\gamma$, IL-17A, TNF- $\alpha$ were undetectable in all pts. IL-1 $\beta$ was elevated in 1 patient only. ADA serum trough levels were in the therapeutic range in $7 / 10$ pts. One patient developed high antidrug antibodies titer and ADA serum trough level of 0 with a concomitant increase in VAS pain and IL-6 concentration. Another patient with low ADA trough levels and no antibodies improved after providing ADA weekly. The disease relapsed in 9/10 pts, within 4-6 weeks following ADA interruption, 7 pts enrolled into the extension study.

\begin{tabular}{lccc}
\hline & Baseline mean (SD) & 24 weeks mean (SD) & $\mathrm{P}$ \\
\hline Swollen joints & $4.6(4.2)$ & $0.6(0.4)$ & 0.006 \\
Tender joints & $19(18.7)$ & $12.6(10.9)$ & Non significant (NS) \\
Physician VAS & $51.5(18.5)$ & $24.5(16)$ & 0.002 \\
Patient pain VAS & $72(19)$ & $56(33)$ & $\mathrm{NS}$ \\
BDCAF & $5.4(1.6)$ & $2.1(1.4)$ & 0.001 \\
HAQ & $1.76(0.8)$ & $1.6(0.9)$ & $\mathrm{NS}$ \\
IL-6 pg/ml & $10.06(13.4)$ & $2.02(0.8)$ & 0.042 \\
\hline
\end{tabular}

Conclusions: ADA treatment was well tolerated and achieved a significant improvement in arthritis and mucocutaneous manifestations and lowered IL-6 serum concentration in all study pts but only $40 \%$ reported significant pain reduction. A subset of pts with insufficient improvement in joint tenderness and generalized pain may require comprehensive pain management besides antiinflammatory therapy.

Acknowledgements: ABBVIE donated the study medication and supported the lab work

Disclosure of Interest: None declared

DOI: 10.1136/annrheumdis-2017-eular.4939

\section{THU0344 A RHEUMATOLOGY LED PATHWAY FOR THE INITIAL MANAGEMENT OF GCA IMPROVES DIAGNOSTIC OUTCOMES COMPARED TO THE GENERALIST}

Z. Farah, M. Gulati, M. Matheou, H. Alam, S. Hamdulay. Rheumatology, Northwick Park Hospital, London, United Kingdom

Background: Giant Cell Arteritis (GCA) is a medical emergency requiring prompt and appropriate management to prevent complications. The British Society of Rheumatology (BSR) has set out guidelines on the appropriate management of suspected GCA targeting both primary and secondary-care physicians. Hospitals increasingly use care-pathways to facilitate appropriate initial management of GCA by non-rheumatologists. Evidence is limited, however, on the impact of such interventions on patient care.

Objectives: To evaluate the impact of designing a GCA care-pathway for nonrheumatologists (acute and general physicians) on patient care in terms of: (i) duration from referral to temporal artery biopsy (TAB) and rheumatology review; (ii) glucocorticoid therapy burden; and (iii) proportion of referrals with a final diagnosis of GCA

Methods: We performed a retrospective study of all patients diagnosed with GCA between 3 periods: prior to introducing a GCA pathway (2007-2009) rheumatology led GCA pathway (2010-2012), and non-rheumatology led GCA pathway (2012-2016). We identified patients from a TAB database and collected general demographic data, initiation of glucocorticoid therapy, referral for TAB and rheumatology clinic, date of TAB and clinic review, biopsy findings, and final diagnosis.

Results: Table 1 summarises the main findings. After introducing the rheumatology-led pathway (2010-2012), rate of referrals for TAB per month declined to 0.78 , the proportion of patients having a TAB within 14 days of referral reached $100 \%$, and the proportion of patients with a positive biopsy increased to $30 \%$ suggesting appropriate use of the pathway and an improvement in care. However introducing a non-rheumatology led GCA pathway (2012-2016), led to increased referral numbers. The proportion of TAB within 14 days decreased, and the proportion with a positive biopsy declined (17\%).

\begin{tabular}{|c|c|c|c|}
\hline & Before GCA Pathway & Rheumatology Led GCA Pathway (1st Cycle) & Non-Rheumatology Led GCA Pathway (2nd Cycle) \\
\hline Dates & Pre-2010 (Jan 07-Sep 09) & 2010 to 2012 (Jan 10-Mar 12) & 2016 (Nov 12-June 16) \\
\hline Study Design & Retrospective & Retrospective & Retrospective \\
\hline Source of Data & Histology database, electronic records & Histology database, electronic records & Histology database, electronic records \\
\hline Number of Patients Biopsied & 54 & 21 & 129 \\
\hline Number of Months & 33 & 27 & 44 \\
\hline Rate of Biopsies (pts/month) & 1.64 & 0.78 & 2.93 \\
\hline Days from referral to TAB; Median (min-max) & $5(0-56)$ & $5(0-13)$ & $9(0-45)$ \\
\hline Proportion with TAB within 14 days & $87 \%$ & $100 \%$ & $66.2 \%$ \\
\hline Proportion with a positive biopsy for GCA & $26 \%$ & $30 \%$ & $17 \%$ \\
\hline Mean Age (years) & 73 & 73 & 71 \\
\hline Gender Ratio (M:F) & $18: 36$ (F 66.7\%) & $4: 17$ (F 80.9\%) & $48: 81(\mathrm{~F} 62.7 \%)$ \\
\hline OUTCOME & GCA Pathway introduced & Pathway continued & Pathway review \\
\hline
\end{tabular}

